\title{
Transconjunctival excision of a conjunctival cyst using computer-assisted 3-D surgical planning in a dog
}

\author{
Jessica Burn ${ }^{1}$, Andras Komaromy ${ }^{1}$, Dodd Sledge ${ }^{2}$, Rebecca Smedley ${ }^{2}$, Sarah Coe ${ }^{2}$, and Sun \\ $\mathrm{Kim}^{3}$ \\ ${ }^{1}$ Michigan State University College of Veterinary Medicine \\ ${ }^{2}$ Michigan State University \\ ${ }^{3}$ Howard Hughes Medical Institute - Purdue University College of Veterinary Medicine
}

March 16, 2021

\begin{abstract}
Investigation of exophthalmos and blood-colored discharge from the left ventral punctum in a dog was consistent with a conjunctival cyst. 3-D prints of the cyst and surrounding facial bones identified a successful transconjunctival approach without an orbitotomy and patency of the left lacrimal duct was re-established.
\end{abstract}

Transconjunctival excision of a conjunctival cyst using computer-assisted 3-D surgical planning in a dog

Jessica B. Burn ${ }^{1}$, András M. Komáromy ${ }^{1}$, Dodd G. Sledge ${ }^{2}$, Rebecca Smedley ${ }^{2}$, Sarah E. Coe ${ }^{2}$, Sun Young $\mathrm{Kim}^{3}$

${ }^{1}$ Department of Small Animal Clinical Sciences, College of Veterinary Medicine, Michigan State University, East Lansing, MI, USA

${ }^{2}$ Diagnostic Center for Population and Animal Health, Michigan State University, Lansing, MI, USA

${ }^{3}$ Department of Veterinary Clinical Sciences, College of Veterinary Medicine, Purdue University, West Lafayette, IN, USA

Address correspondence to Dr. Kim

Department of Veterinary Clinical Sciences

College of Veterinary Medicine, Purdue University

625 Harrison Street, West Lafayette, IN 47907

Phone: $765-494-1107$

Fax: 765-496-1025

Email: kim3315@purdue.edu

Abstract

Investigation of exophthalmos and blood-colored discharge from the left ventral punctum in a dog was consistent with a conjunctival cyst. 3-D prints of the cyst and surrounding facial bones identified a successful transconjunctival approach without an orbitotomy and patency of the left lacrimal duct was re-established. 


\section{Introduction}

Conjunctival cysts in dogs originate from either the conjunctival epithelium or the epithelium of the lacrimal duct and complete excision is typically curative ${ }^{1-3}$. The underlying mechanism of cyst formation remains unclear; however, congenital duct malformation, production of abnormal secretory material, trauma and/or inflammation leading to lacrimal gland hypersecretion and subsequent injury to the walls of the ducts are speculated to lead to passive dilation and cyst formation ${ }^{4-6}$.

Successful medical and surgical treatments for conjunctival cysts include injection of a sclerosing agent such as $1 \%$ polidocanol, surgical marsupialization, and orbitotomy with enucleation to access and surgically excise

the cyst ${ }^{3,7-10}$. Treatment choice is dependent on the size and location of the cyst and the involvement of adjacent anatomic structures. Surgical approaches are often complicated by the presence of the globe itself, the proximity of nerves and large vessels, and the need to work in a small space surrounded by bones and ligaments. This report details the surgical planning and transconjunctival excision of a conjunctival cyst in a dog using a patient-specific 3-D model.

\section{Case history}

A 5-year-old neutered male Cavalier King Charles Spaniel dog presented to the Ophthalmology Service of the College of Veterinary Medicine at Michigan State University for evaluation of dark, blood-colored discharge emanating from the left ventral punctum of eight months duration. Treatment for suspected bilateral allergic conjunctivitis was initiated by the referring veterinarian with neomycin polymyxin B dexamethasone $0.1 \%$ ophthalmic suspension (USP: Bausch and Lomb) twice daily in both eyes for 2 weeks. The dog's right eye responded well, but the left eye remained abnormal.

\section{Initial clinical findings}

Ophthalmic examination yielded a slightly raised left nictitating membrane, mild exophthalmos and lateral deviation of the left globe. Retropulsion of the left globe yielded a mild stream of thick, dark red discharge and fresh blood from the left lower punctum (Figure 1A) Fluorescein passage tests were negative for both nasolacrimal ducts. Normal airflow was present in both nostrils. The remainder of the ophthalmic and physical examination was unremarkable.

\section{Ancillary diagnostic tests}

Computed tomography $(\mathrm{CT})$ of the head revealed a 2-cm diameter, round, well-circumscribed, thin-walled, fluid-filled structure ventromedial to the left globe causing moderate exophthalmos with focal medial displacement and lysis of the osseous orbital wall (Figure 2). Contrast dacrocystorhinography (Omnipaque; iohexol injection 52\%; Marconi Medical Systems) of both nasolacrimal ducts revealed normal dye passage and anatomy. Pooling of dye was observed in the left nasolacrimal duct along the dorsal margin of the mass. Ultrasound-guided fine needle aspiration yielded hemorrhagic fluid with cytologic diagnosis of neutrophilic and macrophagic inflammation and no microorganisms. Aerobic and anaerobic culture of the cyst contents were negative. A presumptive diagnosis of a nasolacrimal cyst was established. Other differential diagnoses that were considered included neoplasia, foreign body or granuloma causing obstruction of the nasolacrimal duct.

\section{Surgical management}

A digital 3-D model of the cystic lesion and its surrounding structures including the globe, bony structures, and the infraorbital artery were segmented and a partial orbitotomy was simulated to expose the cyst using 3-D modeling software (3D Slicer 4.10.2, slicr.org) (Figure 3). The cystic lesion and a portion of skull around the lesion, including the zygomatic, lacrimal, and maxillary bones and their associated foramina, were separately 3-D printed with a $300 \mu \mathrm{m}$ layer thickness (3-D Printer: Form 2 Formlabs). A simulated surgery using the 3-D digital model and manipulation of the 3-D prints established that a transconjunctival approach to the cyst was feasible without an orbitotomy. The 3-D prints were gas sterilized with ethylene oxide for use in surgery. 
The dog was pre-medicated (methadone hydrochloride (Methadone: Bioniche Pharma) $0.5 \mathrm{mg} / \mathrm{kg} \mathrm{IV}$ ), induced (alfaxalone (Alfaxanß, Jurox Animal Health) $1 \mathrm{mg} / \mathrm{kg}$ IV to effect), intubated and maintained on isoflurane gas (Isoflurane: Isothesia, Henry Schein) in oxygen. Maintenance fluids (Lactated Ringer's Injection Rx, USP: Hospira; $5 \mathrm{~mL} / \mathrm{kg} / \mathrm{hr}$ IV) were administered under anesthesia. The left nictitating membrane was protruded and an 8-mm conjunctival incision made on its palpebral side, approximately $10 \mathrm{~mm}$ ventral to the leading edge. The cystic lesion was isolated by surgical dissection, avoiding the angularis oculi vein dorsomedial to the incision, and the deep facial vein, malar artery, infraorbital and maxillary branches of the trigeminal nerve and the buccal branch of the facial nerve ventromedial to the incision. Gentle traction was applied to exteriorize the cyst for further dissection (Figure 1B). A thickened, fibrous band adhered the medial aspect of the cyst capsule to the ventral aspect of the lytic orbital bone; it was transected to allow for removal of the cyst capsule without fragmentation of the bone. The subcutaneous and conjunctival tissues were closed in a simple continuous suture pattern using 6-0 polyglactin 910 (Vicryl: Ethicon). A complete temporary tarsorrhaphy was placed using IV tubing for stents and 4-0 monofilament nylon (Ethilon: Ethicon) to protect the ocular surface. An Elizabethan collar was fitted, and the dog was discharged with oral carprofen (Rimadyl: Pfizer Animal Health) $2.2 \mathrm{mg} / \mathrm{kg}$ twice daily and amoxicillin trihydrate/clavulanate potassium (Clavamox: Pfizer Animal Health) $14.5 \mathrm{mg} / \mathrm{kg}$ twice daily for 2 weeks.

\section{Histopathology}

Histopathologic evaluation of the excised lesion confirmed a benign conjunctival cyst lined by variably eroded to attenuated squamous epithelium with a thick surrounding band of dense collagenous stroma containing occasional perivascular lymphonodular aggregates (Figure 4). Within the cyst lumen and focally within the cyst wall, there was evidence of chronic hemorrhage, including pools of extravasated erythrocytes, aggregates of hemosiderin laden macrophages, and aggregates of mineralized debris. The origin of the cyst could not be confirmed.

\section{Outcome}

The punctal discharge resolved immediately after surgery (Figure 1C). Recheck examination 2 weeks postoperatively confirmed a cosmetic outcome (Figure 1D). The left nasolacrimal duct was patent (positive Jones test) and no complications were noted. Follow-up by telephone conversation eleven months post-operatively confirmed the dog remained asymptomatic with no recurrence of punctal discharge.

\section{Discussion}

The surgical technique and 3-D modelling described herein allowed for a minimally invasive procedure that eliminated the need to sacrifice the globe or to perform an orbitotomy to access the cyst. To the authors' knowledge, only one other report has documented the use of a patient specific 3-D model to aid in planning the surgical approach to a nasolacrimal duct obstruction in a $\operatorname{dog}{ }^{11}$.

Although advanced imaging including dacryocystorhinography and 3-D CT imaging are valuable tools to identify orbital lesions and their surrounding structures and to develop a treatment plan $^{12-15}$, these imaging modalities only provide a digital rendition that cannot be handled or manipulated by the surgeon intraoperatively. In contrast to rendered images, segmented 3-D surface models in this report allowed for digital manipulation of individual anatomical structures with an unlimited number of trials ${ }^{11,16}$. The 3-D printed portion of the skull and of the cyst allowed for pre-operative measurement of the cyst and identification of the best surgical approach. Although the virtual surgery indicated a partial orbitotomy would optimize exposure of the lesion, the tangible 3-D printed models showed the possibility of excision of the cyst with gentle traction via a transconjunctival approach. The 3-D model provided sufficient anatomical detail to explore and remove the cyst while avoiding the deep facial vein, anastomolic branch to the ventral external ophthalmic vein, malar artery, zygomaticofacial nerve, and zygomaticotemporal nerve to minimize the risk of hemorrhage and to preserve normal motor and sensory function. With the degree of lysis present, there was concern for bone fragmentation during removal of the embedded cystic tissue. Therefore, as much of the exposed cystic material as possible was debrided for biopsy, and a portion of a fibrotic band that was attached to the cystic capsule was transected and left adhered to the bone. Histopathological results confirmed a benign 
cyst; as such, no revision surgery was performed to extract the remaining fibrous tissue.

Intralesional treatment with a sclerosing agent such as $1 \%$ polidocanol could have been considered for this case (Zimmerman et al. , 2019); however, the owners elected for surgical excision to maximize the potential for resolution and minimizing the risk of nasolacrimal duct obstruction and blood-colored discharge. Neoplastic disease, such as nasal adenocarcinoma, osteosarcoma, lymphoma, and meningioma were considered as differential diagnoses prior to sampling ${ }^{17}$. In contrast to the marginal excision required for removal of a benign cyst, as was described in this case, a malignant lesion would necessitate orbitotomy or exenteration in an attempt to achieve clean margins.

Streamlining the surgical approach to preserve the globe and bony structures surrounding the globe and only printing the portion of the skull of interest minimized surgical time and the associated anesthetic and surgical costs ${ }^{11}$. A printing time of only 1.5 hours and material costs of approximately $\$ 10$ USD were incurred in this report.

This case report highlights the benefits of CT imaging in concurrence with 3-D surface modelling and printing to facilitate planning a minimally invasive transconjunctival approach to successfully remove a conjunctival cyst, while sparing the globe and other orbital structures. Our surgical outcome was excellent with normal function of the nasolacrimal duct and no postoperative complications.

\section{Disclosure Statement}

The authors declare that they have no potential conflicts of interest with respect to the research, authorship and publication of this article.

Author Contributions:

Jessica B. Burn: Involvement in the clinical case, manuscript preparation and revision, and compilation of figures

András M. Komáromy: Involvement in the clinical case, supportive role in manuscript preparation and revision, formatting of figures

Dodd G. Sledge: Contribution to the histopathology section of the manuscript and provision of a histopathology figure

Rebecca Smedley: Involved in histopathology preparation and analysis

Sarah E. Coe: Involved in histopathology preparation and analysis

Sun Young Kim: Involvement in the clinical case, supportive role in manuscript preparation and revision, provision of $\mathrm{CT}$ images, provision of the 3 -D printed model

\section{References}

1. Delgado E. Dacryops of the lacrimal gland in a dog. Vet Ophthalmol . 2013;16(2):153-158.

2. Lamagna B, Peruccio C, Guardascione A, et al. Conjunctival dacryops in two golden retrievers. Vet Ophthalmol . 2012;15(3):194-199.

3. Barbe C, Raymond-Letron I, Mias G, Charron J, Goulle F. Marsupialization of a cyst of the nictitating membrane in three dogs. Vet Ophthalmol . 2017;20(2):181-188.

4. Grahn BH, Mason RA. Epiphora associated with dacryops in a dog.J Am Anim Hosp Assoc . 1995;31(1):1519.

5. Bullock J. Lacrimal ductal cysts. Ophthalmology . 1986;93(10):1355-1360.

6. Duke-Elder S. The ocular adnexa: Lacrimal, orbital and para-orbital diseases. In: System of Ophthalmology. Henry Kimpton, London . ; 1974:638-643. 
7. Zimmerman KL, Stefanacci JD, Greenberg SM. Use of $1 \%$ polidocanol and pre- and post-computed tomography for treatment of a suspected nasolacrimal duct cyst in a dog. Vet Ophthalmol . 2019;22(3):374380 .

8. Davidson HJ, Blanchard GL. Periorbital epidermoid cyst in the medial canthus of three dogs. $J$ Am Vet Med Assoc . 1991;198(2):271-272.

9. Bodner L, Woldenberg Y, Bar-Ziv J. Radiographic features of large cystic lesions of the jaws in children. Pediatr Radiol . 2003;33(1):3-6.

10. Giudice C, Marco R, Mirko R, Luca M, Giorgio C. Zygomatic gland adenoma in a dog: histochemical and immunohistochemical evaluation. Vet Ophthalmol . 2005;8(1):13-16.

11. Burn JB, Kim SY, Park SA, Komaromy AM, Pirie CG. Use of 3-dimensional printing in surgical exploration of a nasolacrimal duct obstruction in a dog. Can Vet $J$. 2020;61(2):129-134.

12. Ito K, Asano K, Urano T, et al. Periorbital cyst with bone defect in a dog. J Vet Med Sci . 2006;68(7):747748 .

13. Ota J, Pearce JW, Finn MJ, Johnson GC, Giuliano EA. Dacryops (lacrimal cyst) in three young labrador retrievers. J Am Anim Hosp Assoc . 2009;45:191-196.

14. Lussier B, Carrier M. Surgical Treatment of Recurrent Dacryocystitis Secondary to Cystic Dilatation of the Nasolacrimal Duct in a Dog.J Am Anim Hosp Assoc . 2004;40:216-219.

15. Featherstone H, Llabres Diaz F. Maxillary bone epithelial cyst in a dog. J Sm Anim Pr . 2003;44(12):541545 .

16. Lam G, Kim S-Y. Three-dimensional computer-assisted surgical planning and use of three-dimensional printing in the repair of a complex articular femoral fracture in a dog. VCOT Open . 2018;1:e12-e18. doi:10.1055/s-0038-1676062

17. Flaherty EH, Robinson NA, Pizzirani S, Pumphrey SA. Evaluation of cytology and histopathology for the diagnosis of canine orbital neoplasia: 112 cases (2004-2019) and review of the literature. Vet Ophthalmol . 2020;23(2):259-268. doi:10.1111/vop.12717
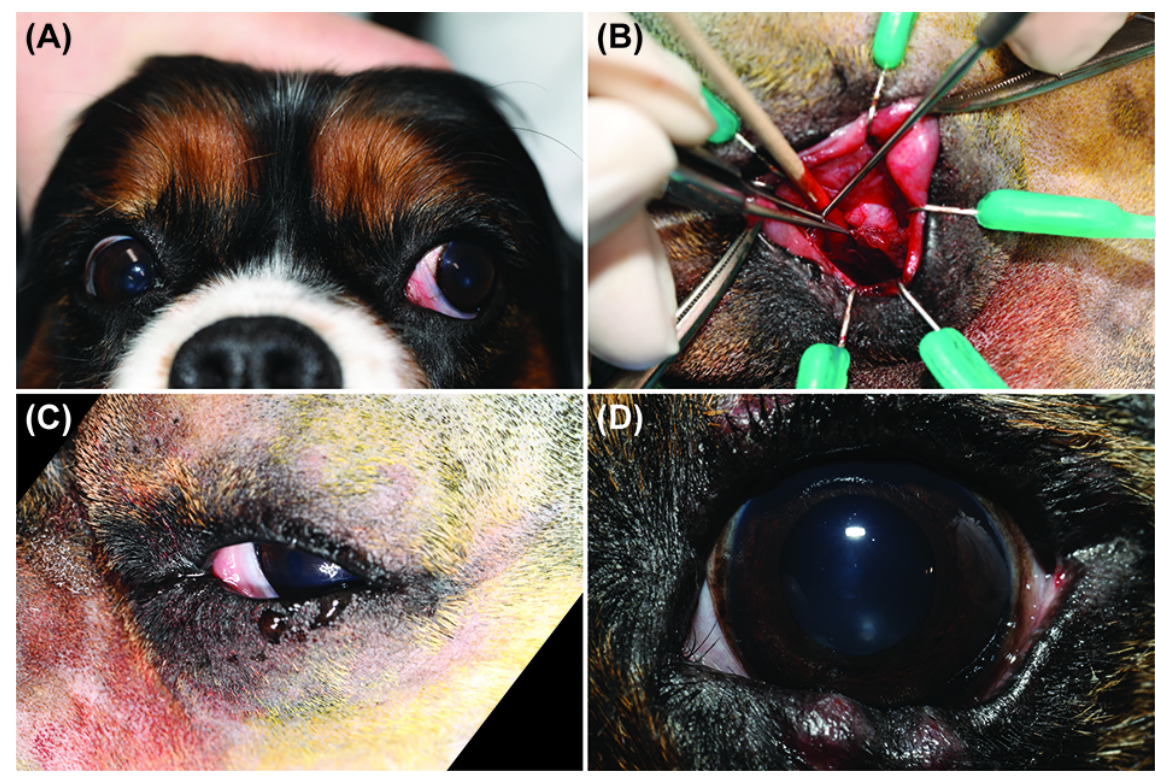

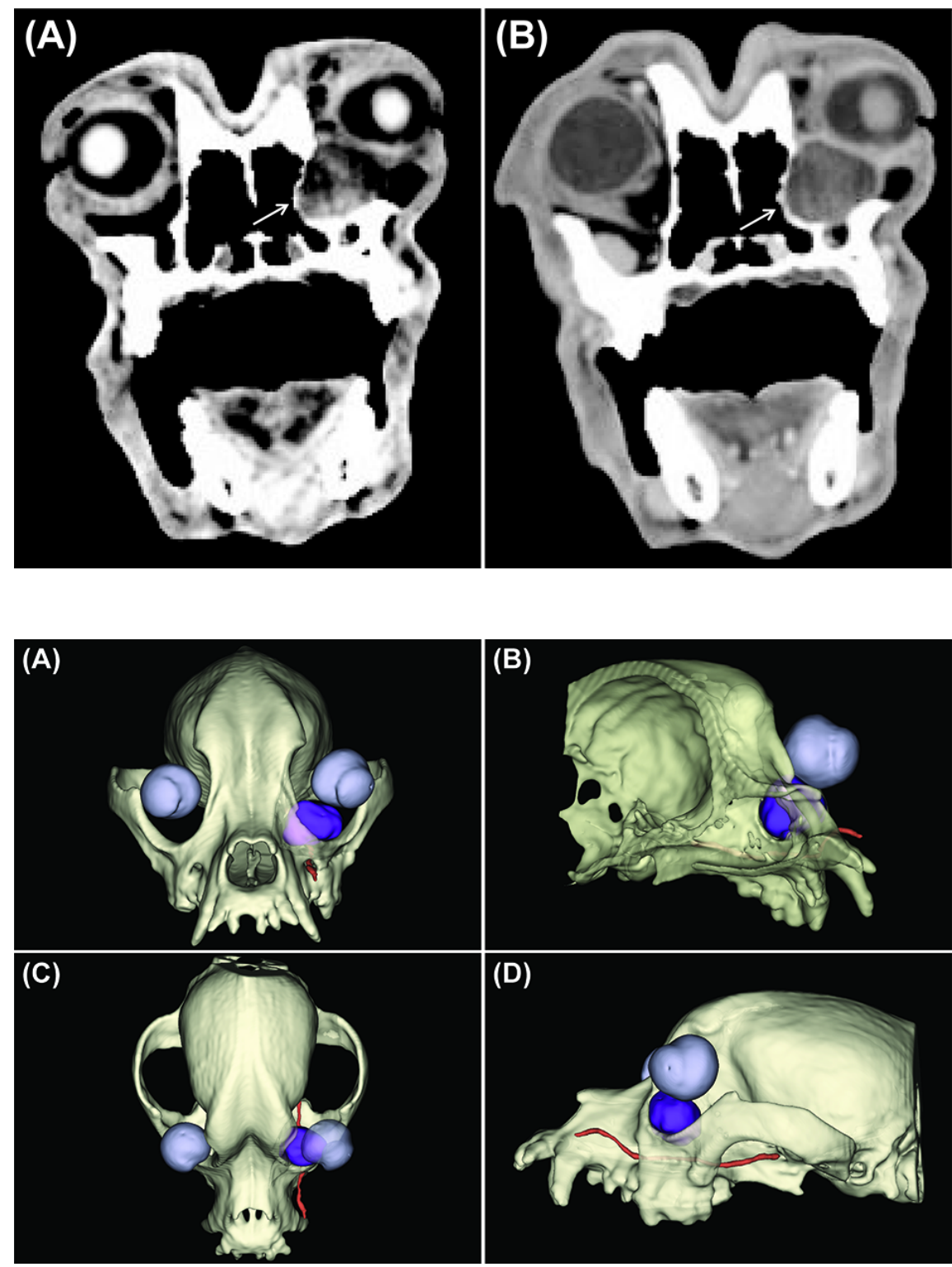
(A)

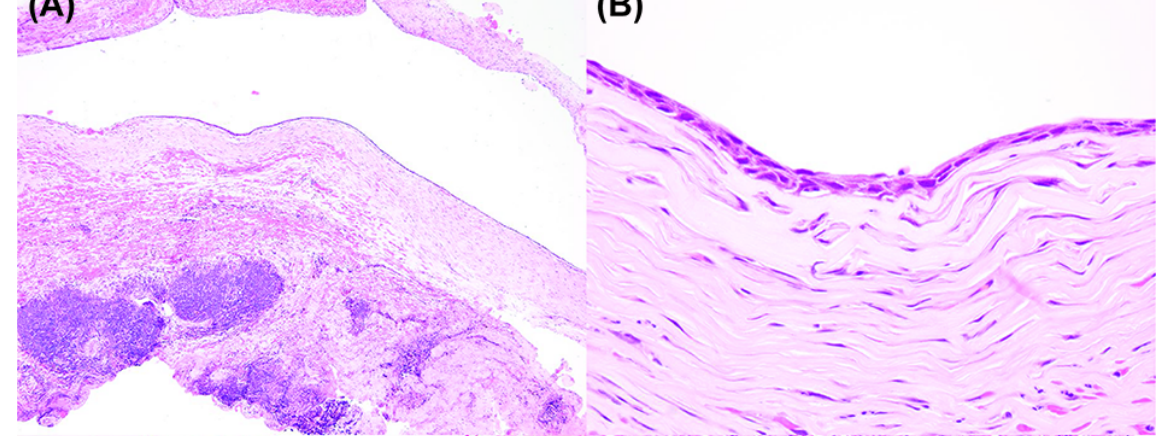

(C)
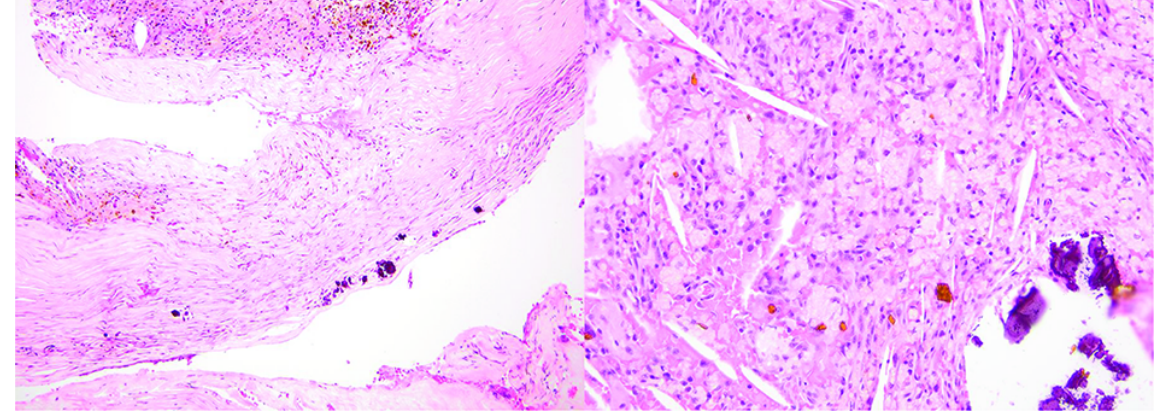